\title{
Design of PM2.5 Detector Based on Internet of Things (IOT) Technology
}

\author{
Li Qiuhua ${ }^{a}$, Hao Chuanzhub \\ Shandong Huayu University Of Technology, East Road of University, Dezhou, 253034, China \\ a lqhlqh000@126.com, ${ }^{b}$ haochuanzhu@163.com
}

Keywords: Internet of Things; PM2.5; PMS5003 sensor; ESP8266

\begin{abstract}
In this paper, the small PM2.5 detector the Internet of Things (IOT) transmission and PM2.5 and temperature and humidity detection software and hardware system are designed. The circuit design of PMS5003 sensor and SI7021 temperature and humidity sensor is carried out by using ESP8266 MCU with WIFI chip uploading method. The PMS5003 sensor collect the concentration value and temperature and humidity value of PM2.5 in the air, the analog voltage signal output by the sensor is converted into a digital signal, and the data is transmitted to the single chip ESP8266. The results of the detection are uploaded to the cloud client. Users can view the data through the mobile phone. When the value of PM2.5 is too high, the fan can be turned on automatically. This paper designs these functional modules. Through further installation and debugging, the function of the whole system can be realized and the purpose of detection can be achieved.
\end{abstract}

\section{Introduction}

With the development of science and technology, many new types of household goods have been developed and manufactured, and the quality of life of people has been greatly improved. At present, air pollution is serious in some areas of our country, and PM2.5 is one of the important factors causing pollution. How to detect the value of PM2.5 in real time and show it through advanced and safe technology, and make a good guarantee and early warning for our daily travel, work and life, is a very practical technical research and development problem. This paper solved the design of the household PM2.5 detection device economically, practically and effectively.

\section{Hardware Design of Household PM2.5 Detector System}

In order to realize the design of PM2.5 concentration detection system, it is necessary to meet the requirement of PM2.5 concentration collection, data signal processing, display the detection results and send them to the mobile phone through wireless transmission[1]. This design is based on the concept of Internet of Things technology, to first select the appropriate transmission method, and then select the appropriate sensor device, display, etc.

\subsection{Data transmission method}

The data transmission method adopts WIFI wireless transmission. The main principle is to group the information sent by the user, and then convert the group information into microwave signals to realize various functions of the wireless local area network. These data transmission methods have many advantages, including long transmission distance and large coverage; high transmission rate with speed up to $11 \mathrm{M}$; the system capacity meets requirements, suitable for continuous transmission of big data; with high security, it can support WAPI/WEP/WPA/WPA2 security standard, and it can be protected by configuring different security standards; no additional overhead for transmitting data can be achieved through a household wireless router, which is economical.

\subsection{Selection of main controller}

The main controller is controlled by ESP8266 single chip microcomputer. It has the characteristics of small size, light weight, low power consumption, strong function and high cost 
performance. It plays an overall role in the whole system. It can control and receive all kinds of parameters of the sensor. At the same time, it can transmit data through WIFI and display relevant data through liquid crystal to complete the transmission sensors and blower to control their functions. ESP8266 has its own Wi-Fi chip, which can complete wireless transmission independently and completely. In addition, ESP8266 can be used as Wi-Fi adapter only through SPI / SDIO interface and can be applied to any microcontroller-based design. ESP8266 has 32 pins, and the input/output ports are GPIO0, GPIO2, and GPIO4[2], shown in Figure 1.

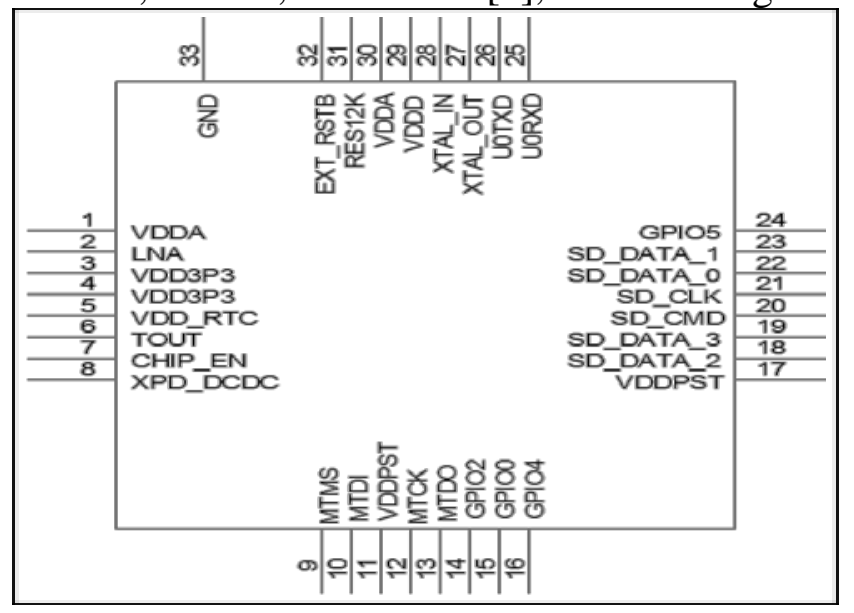

Figure 1 Single chip ESP8266 pin distribution diagram.

ESP8266 WIFI chip transmits data by subscription. The chip collects large data through sensors. The cloud selects the content of the data and transmits it to the chip. ESP8266 only transmits the selected data to the client. This transmission mode can greatly increase the speed of data transmission, so that the main data can be received and sent in real time.

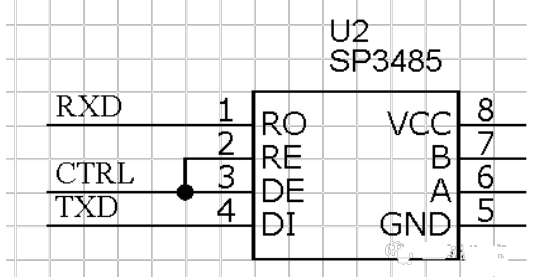

Figure 2 PMS5003 sensor serial port diagram

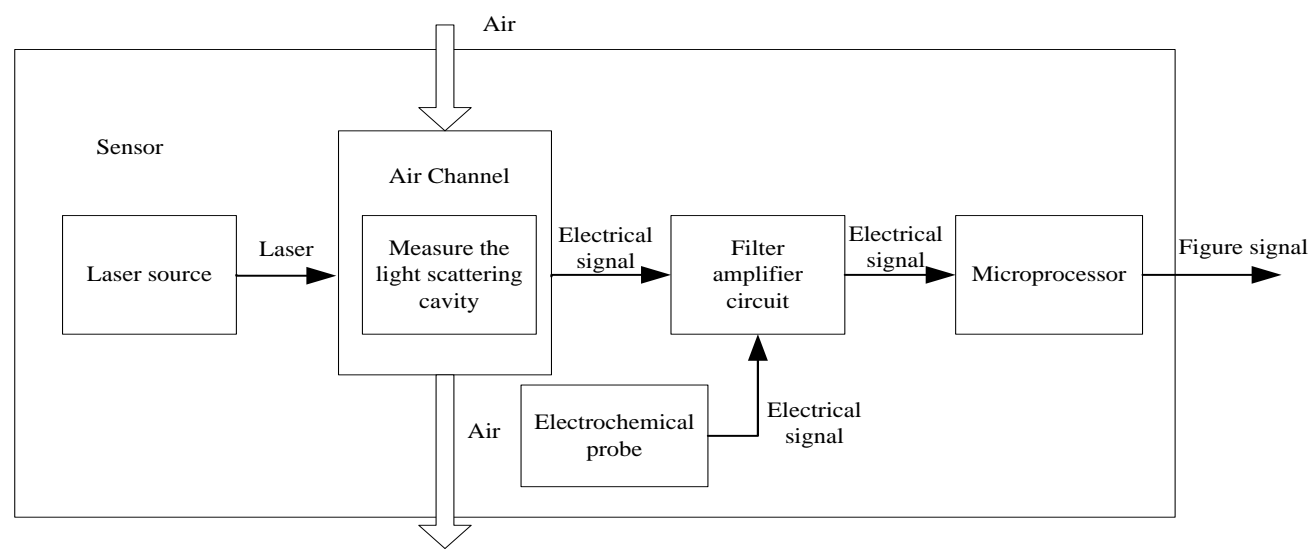

Figure 3 The working flow chart of PMS5003 sensor

\subsection{Selection of PM2.5 detection sensor}

The PM2.5 detection sensor uses the PMS5003 sensor, shown in Figure 2 and Figure 3, which uses laser scattering to collect data, which is scattered by the built-in laser to illuminate the suspended matter in the air, and collect the scattered light at a special angle, which is a function of the scattered light as a function of time. The microprocessor calculates the amount of particulate 
matter per unit volume. The sensor adopts serial communication mode, the first leg is connected to the serial port input of TXD, and the second leg is connected to the serial port output of RXD. When the ESP8266 send data, the PM2.5 data is sent through the TXD. The control CTRL is high level, when the ESP8266 wants to receive data, PM2.5 data is received through RXD. The control CTRL is low level.

\subsection{The module design of temperature and humidity sensor}

The type of temperature and humidity sensor is SI7021 temperature and humidity sensor, which mainly collects the temperature and humidity value and outputs a digital quantity and uploads it to the WIFI data terminal. The module of the temperature and humidity sensor is connected to the IIC interface of the ESP8266 through a single bus[3]. The connection diagram is shown in Figure 4.

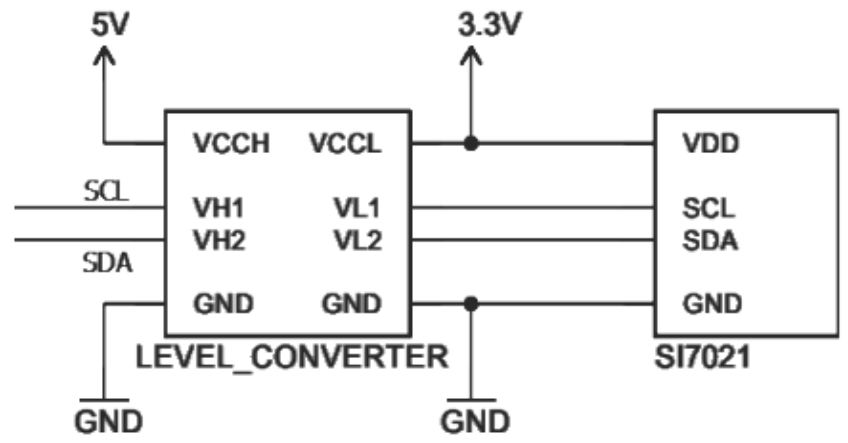

Figure 4 The connection diagram of temperature and humidity sensor

\subsection{The lectotype of fan and its relay}

The fan pipe adopts Hongfeng round small pipe. With low heat consumption, it can increase the efficiency of use by 3 to 4 times, with small size, it is convenient to install and use, and with low noise, it does not affect normal family life when opened. It has a long service life and can be used for about 7 to 9 years. The fan motor is a $220 \mathrm{~V}$ double ball high speed motor with input power of $100 \mathrm{~W}$ and frequency of $50 \mathrm{HZ}$. It can generate 2100 square meters of air flow per hour, which can meet the needs of most families. The fan control module uses a single chip microcomputer which is powered by $3.3 \mathrm{~V}$, and the $220 \mathrm{~V}$ voltage is controlled by a relay for the work, to select and use one way relay[4]. The relay circuit diagram is shown in Figure 5.

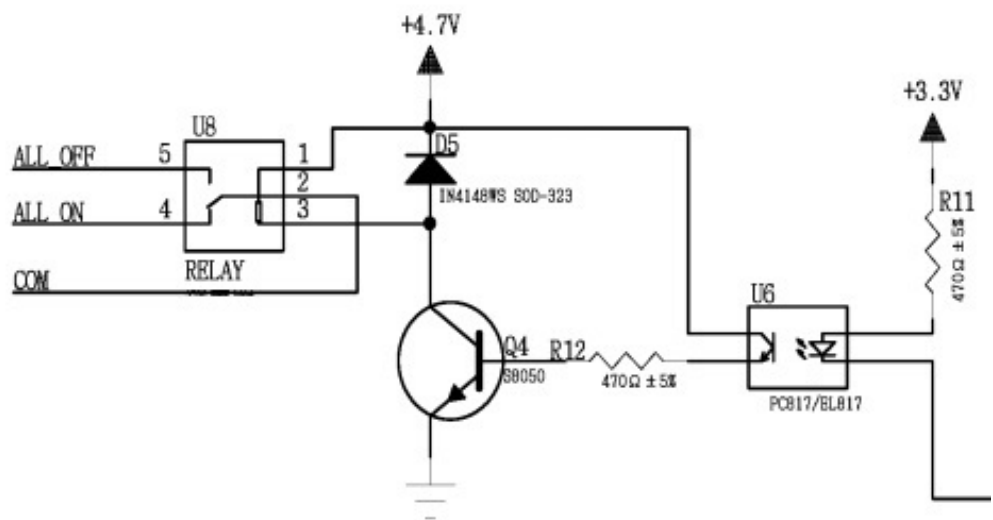

Figure 5 Relay circuit diagram.

\section{The Software Design of Household PM2.5 Detector System}

The software function should realize the host board ESP8266 to accept and process the data of each sensor, and then send the data to the cloud server, and realize the automatic detection, transmission and the programming viewed by mobile phone. The process flow diagram is shown in Figure 6 . The temperature and humidity sensor control adopts the form of subroutine, which packs 
the collected parameters of the temperature and humidity sensor into a library and brings into the subroutine in the design of the general program. The PMS5003 sensor program uses the same method as the temperature and humidity sensor to realize the data collection and transmission instruction of the sensor[5][6].

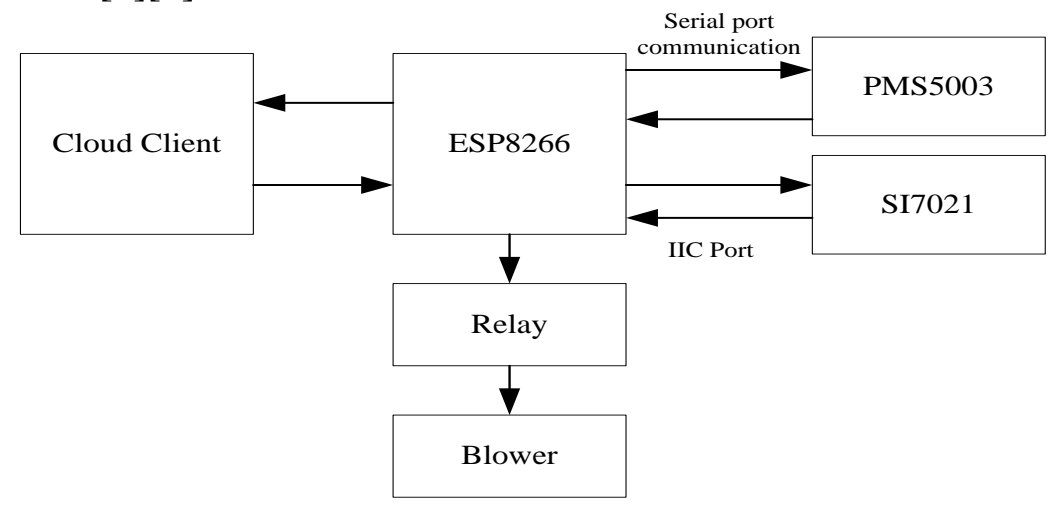

Figure 6 System working flow chart

\section{Conclusion}

This design realizes the detection and transmission of PM2.5 concentration through the combination of ESP8266 and sensor technology. ESP8266 chip is the control center. PMS5003 sensor collects particulate matter concentration. SI7021 sensor collects temperature and humidity signal. ESP8266 chip uploads program, and Arduino programming upload, mobile downloads of cloud apps to remind people to take appropriate safety precautions to improve the current environment. The test system designed is simple and practical, which is suitable for the public's need of PM2.5 concentration detection. The final device is made of inexpensive materials and it is simple to carry.

\section{References}

[1] Ning A M, Wen J H, Zheng D Z, et al. Advances in monitoring technologies and its comparison research for PM2.5[J]. Metrology \& Measurement Technology, 2013, 33(4): 11-14.

[2 ] Lee H J, Coull B A, Bell M L, et al. Use of satellite-based aerosol optical depth and spatial clustering to predict ambient PM 2.5, concentrations[J]. Environmental Research, 2012, 118(118):8-15.

[3] Machtei Eli E, Zigdon Hadar, Levin, Liran, Peled Micha. Novel ultrasotic device to the distance from the bottom of the osteotome to various anatomic landmarks. [J].Journal of Periodontology, 2010,817 .

[4] V.A.Barkhatov. Normalizing ultrasonic pulses in time-delay and distance measuring systems [J]. Russian Journal of Nondestructive Testing, 2009,456.

[5] Gu H H, Wang K P, Wang C F, et al. Household PM2.5 detector[J]. Silicon Valley, 2015(2):11-11.

[6] Wang H, Kong L R. Design of a Wireless PM2. 5 Concentration Detection System [J]. Electronic Science and Technology, 2015, 28(7):133-136. 